\title{
Semiotic foundations of logic
}

VLADIMIR I. SHALACK

\begin{abstract}
The article offers a look at the combinatorial logic as the logic of signs operating in the most general sense. For this it is proposed slightly reformulate it in terms of introducing and replacement of the definitions.
\end{abstract}

Keywords: combinatory logic, semiotics, definition, logic foundations

\section{Language selection}

Let's imagine for a moment what would be like the classical logic, if we had not studied it in the language of negation, conjunction, disjunction and implication, but in the language of the Sheffer stroke. I recall that it can be defined with help of negation and conjunction as follows

$$
A \mid B={ }_{D f} \neg(A \wedge B) .
$$

In turn, all connectives can be defined with help of the Sheffer stroke in following manner

$$
\begin{gathered}
\neg A={ }_{D f}(A \mid A) \\
A \wedge B={ }_{D f}(A \mid B) \mid(A \mid B) \\
A \vee B={ }_{D f}(A \mid A) \mid(B \mid B) \\
A \supset B={ }_{D f} A \mid(B \mid B) .
\end{gathered}
$$

Modus ponens rule takes the following form

$$
\frac{A, A \mid(B \mid B)}{B} .
$$


We can go further and following the ideas of M. Schönfinkel to define two-argument infix quantifier ' $\mid x$,

$$
\left.A\right|^{x} B={ }_{D f} \forall x(A \mid B) .
$$

Now we can use it to define Sheffer stroke and quantifiers.

$A\left|B={ }_{D f} A\right|^{x} B$ where the variable $x$ is not free in the formulas $A$ and $B$;

$\forall x A=\left.{ }_{D f}\left(\left.A\right|^{y} A\right)\right|^{x}\left(\left.A\right|^{y} A\right)$ where the variable $y$ is not free in the formula $A$;

$\exists x A=\left.{ }_{D f}\left(\left.A\right|^{x} A\right)\right|^{y}\left(\left.A\right|^{x} A\right)$ where the variable $y$ is not free in the formula $A$.

The rule for the introduction of the universal quantifier takes the form

$$
\frac{\vdash A}{\left.\vdash\left(\left.A\right|^{y} A\right)\right|^{x}\left(\left.A\right|^{y} A\right)} .
$$

The language containing the only quantifier ' $x$ ' is functionally complete, and has the same expressive power as the language of the classical predicate logic.

Imagine now that theorems of Principia Mathematica are formulated and proved in this language, and that all the fundamental theorems of logic, arithmetic and set theory are described in the language.

We would have the same results as today, but it would be difficult to convince other people that what we learn is really logic. In response, we probably would have heard that we have created an interesting mathematical tool, but it has little to do with logic.

\section{Combinatory logic}

Something similar has happened to the combinatory logic, which was born December 7, 1920, when M. Schönfinkel has made his now famous report to the Mathematical Society of Göttingen. In this report Schönfinkel [4] has showed that not only logical connectives but also quantifiers can be reduced to a single two-place operation. 
He also showed that under the assumption that functions themselves can serve as arguments to other functions and to be their values, we can get along one-argument functions, and two operations to combine them, which can be summarized as follows

$$
\mathbf{K} x y=x \text { and } \mathbf{S} x y z=x z(y z) .
$$

The role of $\mathbf{K}$ and $\mathbf{S}$ in the language is similar to that of the connectives in the logic, but the fundamental difference is that they are applicable to expressions that represent the objects of any nature (!) rather than for solely sentences.

With these operations, which are called combinators, we can define any other operations with functions including quantifier ' $\mid x$ '. In this sense, the set of $\mathbf{K}$ and $\mathbf{S}$ is complete. The theory of combinators is called combinatory logic.

\section{Alphabet}

1. Var - a set of variables;

2. $\mathbf{K}$ and $\mathbf{S}-$ constants;

3. ), ( - brackets;

4. $\geqslant-$ reduction character.

\section{Terms}

1. All $x \in V$ Var are terms;

2. $\mathbf{K}$ and $\mathbf{S}$ are terms;

3. If $X$ and $Y$ are terms, then $(X Y)$ is a term;

4. Nothing else is a term.

\section{Reductions}

1. If $X$ and $Y$ are terms, then $X \geqslant Y$ is reduction; 
2. Nothing else is reduction.

\section{Axioms}

A. $1 X \geqslant X$

A. $2 \mathbf{K} X Y \geqslant X$

A.3 $\mathbf{S} X Y Z \geqslant X Z(Y Z)$

\section{Rules}

$$
\begin{aligned}
& \text { R. } 1 X \geqslant Y \Longrightarrow X Z \geqslant Y Z \\
& \text { R. } 2 X \geqslant Y \Longrightarrow Z X \geqslant Z Y \\
& \text { R. } 3 X \geqslant Y, Y \geqslant Z \Longrightarrow X \geqslant Z
\end{aligned}
$$

We are not going to develop in detail the combinatory logic and prove metatheorems related to it.

Unfortunately, due to the high degree of abstraction of the combinatory logic, it is not widespread, although many logicians have heard of its existence. The combinatory logic is much more known to specialists in computer science, which refer to it as a mathematical apparatus of functional programming. It is not considered as a theory of correct reasoning. It seems unclear how to use it to analyze usual reasoning. There were many attempts to synthesize the combinatory logic with the logic in the traditional sense, but as a result received either contradictory logical systems, or systems that have not received wide acceptance and recognition.

\section{The fundamental nature of the combinatory logic}

The main obstacle to widespread use of the combinatory logic to analyze the reasoning lies in the highly abstract nature of the basic combinators, and hence the lack of understanding of how to make their use in natural discourse. The situation is somewhat similar to that if we developed a logic-based language with the single Sheffer stroke. 
However, the combinatory logic is self-sufficient. There is no need to go beyond it, to present it as the logic of constructing arguments. It's enough just to reformulate it a bit.

First of all it is necessary to give up some of the stereotypes. For a long time it was a stereotype of logic as a theory about relationships in the sphere of common terms. It was a characteristic of the Aristotelian approach, which has dominated for over two thousand years. G. Frege has refused the stereotype and begun to examine the logic as a theory of propositional functions. According to his words, the logic is a theory of Truth Being.

This view turned out to be very fruitful, and we still are under its influence. The theory of combinators does not fit neither Aristotlian, nor Fregean approach, since appeals to a more fundamental entities than the common terms and propositions. It seems to me, in this case we are dealing with the logic of signs operating in the most general sense.

'Combinatory logic is a branch of mathematical logic which concerns itself with the ultimate foundations. Its purpose is the analysis of certain notions of such basic character that they are ordinary taken for granted' [3, p. 1].

The expressions of language, considered as signs, act as representatives of the various objects of thought, which can be things, properties, functions, relationships, etc. Assignment of thought objects to the specific categories is possible, but it isn't a problem of logic, since it can happen only as a result of later cognitive activity of the subject of cognition. The logic is a servant of science, not trendsetter.

The power of language as an instrument of cognition is that it allows us to manipulate objects of extralinguistic reality on the sign level. This manipulation can be represented as a chain of transitions from one signs to another. In the process of manipulating signs the relationship between them and the objects of external reality should not be lost. This is a necessary condition for the correctness of our cognitive activity. Some transitions between signs can be justified by the already known properties of the objects of thought correlated to them. Other transitions are due to study the intrinsic properties of 
sign systems. In a sufficiently general form the consequence relation between signs can be defined as follows:

$U$ follows from the premisses $\Sigma=\left\{V_{1}, \ldots, V_{n}\right\}$, if and only if there exists a rule $R$, which allows on the basis of the values of premises $\Sigma$ to determine the value of the expression $U$.

Formulated in this way the idea of reasoning does not need to clarify what specific linguistic expressions are used, what is the nature of the correspondence between these expressions and extralinguistic reality, what exactly is this reality.

\section{What is the rule $R$ ?}

In the classical logic, where the premises and conclusion are sentences, this rule is specified as 'if the premises $V_{1}, \ldots, V_{n}$ are true, then the conclusion $U$ is true'. Obviously, in this case, the rule $R$ is a partial function that is defined only when all the premises are true. If at least one of the premises is false, we can not say with certainty what will be the truth value of the conclusion. In this case the problem is complicated by the fact that for sufficiently rich theory it is fundamentally impossible to prove that the theory is consistent, and to prove that there is at least one model that makes all the premises true. The standard definition of the semantic consequence stops working.

Our definition does not require that all the premises were true. We can build a system of reasoning in which the sentence not- $A$ follows from the sentence $A$. Indeed, if we know the truth-value of the proposition $A$, then there is no problem to find the truth-value of proposition not- $A$. When you're trying to explain it to professional logician, you will often come across a misunderstanding and opposition, the reason of which are stereotypes inherited from the classical logic approach.

Our definition does not demand that assumptions and conclusions necessarily have been sentences. If you take the language of arithmetic, the formula $t_{1}=t_{2}$ follows from two terms $t_{1}$ and $t_{2}$, because, knowing the values of arithmetic terms $t_{1}$ and $t_{2}$, we can always determine the truth value of the formula $t_{1}=t_{2}$. Similarly, the 
term $t_{2}$ follows from the terms $t_{1}$ and $t_{1}+t_{2}$. In the last example we have the consequence relation between the terms, not sentences.

Inference rules of any system of logic, that we use during construction of the syntactic metalanguage proofs, are also examples of rule $R$ from our definitions of consequence.

The rule $R$ can be a computer program, that from the input parameters (premisses) calculates the result (conclusion), which can be a solution of the differential equation, a corrected word from a text, an informative chart, a piece of music, characters that appear on the screen depending on what keys are pressed, etc.

A student of Architectural Institute in defending diploma must convince the commission of examiners that if you bring sand, water, cement and bricks, and then follow the course of action to certain rules and drawings of the project, the result will really skyscraper, and not another wreck.

A person who is engaged in guesswork, also follows the rules associated with some sign system. Astrologers, African shamans, fortune-tellers on the cards use their sign systems and their own rules relating assumptions and conclusions.

In natural language sentence 'Mary loves Bob' follows from the words 'Bob', 'Mary', 'love'. This following takes place quite independently of whether or not Mary loves Bob. It takes place because of our knowledge of the rules of morphology, grammar and semantics of English. Even if we are for the first time in our lives hear or read this sentence, but if the values of the words 'Bob', 'Mary' and 'love' are known to us, thanks to our knowledge of the rules of language, we always can define the value of the sentence 'Mary loves Bob'.

\section{$5 \quad$ Formalism of signs}

As can be seen from the above examples our definition covers a fairly wide range of phenomena. It is not limited to sentences, but is applicable to the signs of a different nature. In order to cover it in one logical formalism, we must find a starting point to represent the signs.

To do this, I recall the words of a well-known linguist Emile Benveniste that '. . language has a configuration in all its parts and as a totality. It is in addition organised as an arrangement of distinct 
and distinguishing 'signs', capable themselves of being broken down into inferior units or of being grouped into complex units. This great structure, which includes substructures of several levels, gives its form to content of thought. . Linguistic form is not only the condition for transmissibility, but first of all the condition for the realization of thought. We do not grasp thought unless it has already been adapted to the framework of language' [1, pp. 55-56].

The basic idea is that from the syntactic point of view signs form a hierarchy. Complex symbols are obtained by combining simpler ones. To do this, we can use a number of different brackets.

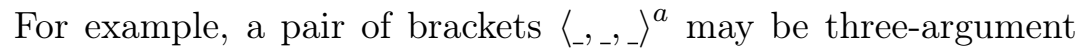
operation, which allows us to construct complex sign 〈'apple', ' $i s$ ', 'red' $\rangle^{a}$ from three words 'apple', 'is' and 'red'. From signs 'sky', ' $i s$ ' and 'blue' we can construct a new sign $\langle\text { 'sky', 'is', 'blue' }\rangle^{a}$. Other types of brackets will be needed to build such signs as $\langle$ ' + ', ' 3 ', '2' $\rangle^{b}$ and $\langle\text { 'young', 'man' }\rangle^{c}$.

It is easy to show that in fact we need only one pair of brackets, which is applicable only to pairs of signs. That's how we do that, because it is convenient for the demonstration of connection with the combinatory logic, but application tasks may require different sets of brackets [5].

\section{Alphabet}

1. Var - a set of variables.

2. Const - possibly empty set of constants;

3. $),(-$ brackets.

\section{Terms}

1. 1. All $x \in \operatorname{Var}$ are terms;

2. All $c \in$ Const are terms;

3. If $U$ and $V$ are terms, then $(U V)$ is term;

4. Nothing else is a term. 
For dropping brackets we accept an agreement about their association to the left.

From the algebraic point of view the models of this language are groupoids, which can be represented as follows

$$
\mathbf{M}=\langle D, O, I\rangle
$$

where $D$ is nonempty set

$O: D \times D \rightarrow D$ - two-argument operation on $D$

$I-$ a function for the interpretation of language constants.

Let $V a l=D^{V a r}-$ the set of all functions assigning values to variables. Then the value of the term $U$ in the model $\mathbf{M}=\langle D, O, I\rangle$ for the evaluation $v \in V a l$ is defined in the obvious way:

1. $|x|_{v}=v(x)$, if $x \in \operatorname{Var}$;

2. $|c|_{v}=I(c)$, if $c \in$ Const;

3. $|(U V)|_{v}=O\left(|U|_{v},|V|_{v}\right)$.

Now we are ready to define rigorously the central concept of consequence $\Sigma \|=U$, where $\Sigma$ is a finite set of terms, and $U$ is a term.

Term $U$ follows from the set of terms $\Sigma=\left\{V_{1}, \ldots, V_{n}\right\}$ if and only if for any model $\boldsymbol{M}$ there exists a function $f$, such that for every valuation $v \in$ Val holds $|U|_{v}=f\left(\left|V_{1}\right|_{v}, \ldots,\left|V_{n}\right|_{v}\right)$.

$$
\left\{V_{1}, \ldots, V_{n}\right\} \|=U \Longleftrightarrow \forall M \exists f \forall v\left(|U|_{v}=f\left(\left|V_{1}\right|_{v}, \ldots,\left|V_{n}\right|_{v}\right)\right)
$$

It is easy to verify that this consequence relation satisfies the well-known conditions of Tarski:

1. If $U \in \Sigma$, then $\Sigma \|=U$;

2. If $\Sigma \|=U$ and $\Sigma \subseteq \Delta$, then $\Sigma \|=U$;

3. If $\Sigma \|=V$ and $\Sigma, V \|=U$, then $\Sigma \|=U$. 
In addition, the defined relation is structural, i.e. $e(\Sigma) \|=e(U)$ follows from $\Sigma \|=U$, where $e-$ is a substitution on set of terms of the language. It means that the consequence relation defines the logic in the sense of Tarski.

Let's turn to the typology of signs of Peirce-Morris. There are three groups of signs.

The first group - signs-indices, whose connection with the signified objects may be due to temporal, spatial and causal types of relationships. Analysis of specific types of relationships is beyond the scope of logic. Distinguishing feature of signs-indices is that they have no significant similarity with their objects, that they refer to individual things, to individual objects, to single sets of objects and direct our attention to their objects by blind compulsion. We can assume that in our formalism signs-indices are among initial constants of language.

The second group of signs - iconic signs. They are linked to the signified objects through the structural similarity. Charles S. Peirce believed that any algebraic equation is an iconic sign because it shows using algebraic symbols (which themselves are not iconic signs) the relationships between it's variables. Every formula of logic and every term can also be regarded as iconic signs. It is thanks to knowledge of their structure we have the ability to operate with them on the basis of formal rules. The situational logics explicitly use iconicity property of complex expressions of the language.

The third group of signs are signs-symbols, whose connection with the signified objects is quite arbitrary, it exists only for the language interpreter. By his request, he gives the role of some objects to be representatives of other objects. In logic the operation of introducing of signs-symbols is well-known and is called the operation of definition. In our language we can represent it as follows.

Suppose that we have in our language a term $T$, all the variables of which are contained among set of variables $\left\{x_{1}, \ldots, x_{n}\right\}$. Then we can add to our language a new constant $\mathbf{D}$, taking the following definition

$$
\mathbf{D} x_{1} \ldots x_{n}={ }_{\text {def }} T \text {. }
$$


By adopting a definition the interpreter is able at any moment to take advantage of the inverse operation of substitution, or disclosure definition, and make the transition from the term $X\left\{\mathbf{D} Z_{1} \ldots Z_{n}\right\}$, in which there is occurrence of the term $\mathbf{D} Z_{1} \ldots Z_{n}$, to the term $X\left\{T\left[Z_{1} / x_{1}, \ldots, Z_{n} / x_{n}\right]\right\}$, obtained as a result of replacement $\mathbf{D} Z_{1} \ldots Z_{n}$ with simultaneous substitution of terms $Z_{1}, \ldots, Z_{n}$ in the term $T$ instead of the variables $x_{1}, \ldots, x_{n}$. We can summarize this as the next rule

$$
\frac{\mathbf{D} x_{1} \ldots x_{n}={ }_{\text {def }} T, \quad X\left\{\mathbf{D} Z_{1} \ldots Z_{n}\right\}}{X\left\{T\left[Z_{1} / x_{1}, \ldots, Z_{n} / x_{n}\right]\right\}} .
$$

The reasoning in this logic can be defined as a sequence of terms $T_{1}, \ldots, T_{n}$, each of which is either one of the premises, or obtained from the previous terms of the sequence by the rule of disclosure of definitions. We specially focus attention on the fact that the possible values of terms occurring in the derivation can be any objects, and not necessarily the truth values of sentences.

Basically, nothing new compared to existing combinatory logic has been proposed. In the combinatory logic we accept reductions $\mathbf{K} X Y \geqslant X$ and $\mathbf{S} X Y Z \geqslant X Z(Y Z)$ as initial, and in our proposed formalism we can introduce definitions $\mathbf{K} X Y=_{\text {def }} X$ and $\mathbf{S} X Y Z=_{\text {def }} X Z(Y Z)$ and get all the same. In this sense, the combinatory logic is embedded in the formalism we have constructed.

\section{Comments}

I. Prior to emergence of the science of logic, much attention was paid to the special operation on linguistic expressions, which later became known as the 'operation of definition'. The idea of this operation is transparent to the understanding and hardly anyone would reject it. You may recall that science begins not so much with theorem proving, as with search for definitions of the various objects and phenomena of reality. Plato's Socrates seeks definitions of beautiful jug, a beautiful woman, beauty by itself, courage, and many others. Thus he extended the language for the description of the world, which allowed him a new way to classify phenomena, to move to a higher level of abstraction and then to think in a new system of concepts. Modern science is unthinkable without an 
abstract and ideal objects, introduced by different kinds of definitions. Any system of axioms implicitly defines the terms imposed by it. The combinatory logic can be rigorously represented as a system of reasoning based on accepted definitions. In this case, its meaning is extremely clear. It is interesting that the initial set of constants Const may be empty, but it can not prevent a logical subject to start using the operation of definition and thus start forming a system of concepts, which are essentially a priori.

II. Initial Schönfinkel's combinators $\mathbf{K}$ and $\mathbf{S}$ are of interest because they represent the complete set of combinators, through which you can define any other combinators. But in the logic of reasoning based on the principle of introducing definitions and their replacement there is no need to declare some initial basic combinators. Through definitions, depending on a specific problem to be solved, you can introduce any desired combinators, not reduce them to a mandatory basis. This significantly simplifies many of constructions and reasoning, making them closer to the natural.

III. It is known that in the combinatorial logic it is provable theorem that each combinator $\mathbf{C}$ has a fixed point, i.e. there exists such a term $T$ that $\mathbf{C} T=T$ is provable. Similarly, we can show that fixed-point definitions are allowed in the proposed formalism. That is, if there exists a term $T$, all the variables of which are contained in set of variables $\left\{y, x_{1}, \ldots, x_{n}\right\}$, then we can introduce the fixed-point definition

$$
\mathbf{D} x_{1} \ldots x_{n}={ }_{d f p} T[\mathbf{D} / y]
$$

These definitions give rise to a conservative extensions of the existing formalism, which at the same time are not inessential. This means that the system of logic, based on the principle of introducing definitions and their replacement which is supplemented by the fixed point definitions, has a greater expressive power than the combinatory logic, since combinatory logic can be embedded into it, but reverse isn't true. It is appropriate to draw an analogy between the constants, introduced by means of fixed point definitions, and $\varepsilon$-terms in the classical logic. 
IV. It is known that all recursive functions can be represented in combinatory logic. They are also obviously representable in the proposed formalism. But the heart of this formalism is the logical operation of definition. Hence it follows that nature of computable functions is exclusively logical and does not depend on adoption any assumptions about the world around us. Since the proposed formalism has clear semiotic foundations and at the same time is closely connected with computability, it can be regarded as the perfect language, which G. Chaitin dreamed of [2].

\section{References}

[1] Benveniste, E., Problems in General Linguistics. University of Miami, 1971. $317 \mathrm{p}$.

[2] Chaitin, G., The Search for the Perfect Language. 2009. http://www . umcs.maine.edu/ ${ }^{\sim}$ chaitin/pi.html

[3] Curry, H. B., Feys, R., Combinatory Logic. V.1. Amsterdam, 1958. $417 \mathrm{p}$.

[4] SchÖnfinkel, M., On the building blocks of mathematical logic, in J. van Heijenoort (eds.), From Frege to Godel, Harvard University Press, 1967, pp. 355-366.

[5] Shalack, V. I., Protologic: A new look at the nature of logic. Doctoral dissertation. 2010. 274 p. 